\title{
Generation of 100-MeV Attosecond Electron Bunches with Terawatt Few-Cycle Laser Pulses
}

\author{
Xing-Long Zhu $\odot,{ }^{1,2,3,4, *}{ }^{*}+$ Wei-Yuan Liu $\odot,{ }^{1,3,+}$ Min Chen $\odot,{ }^{1,3}$ Su-Ming Weng $\odot,{ }^{1,3}$ Feng He, ${ }^{1,3}$ \\ Ralph Assmann, ${ }^{5}$ Zheng-Ming Sheng $\odot,{ }^{1,2,3,4,6, \dagger}$ and Jie Zhang ${ }^{1,3,7}$ \\ ${ }^{1}$ Key Laboratory for Laser Plasmas (MOE), School of Physics and Astronomy, Shanghai Jiao Tong University, \\ Shanghai 200240, China \\ ${ }^{2}$ SUPA, Department of Physics, University of Strathclyde, Glasgow G4 0NG, United Kingdom \\ ${ }^{3}$ Collaborative Innovation Center of IFSA, Shanghai Jiao Tong University, Shanghai 200240, China \\ ${ }^{4}$ Tsung-Dao Lee Institute, Shanghai 200240, China \\ ${ }^{5}$ Deutsches Elektronen-Synchrotron DESY, D-22607 Hamburg, Germany \\ ${ }^{6}$ Cockcroft Institute, Sci-Tech Daresbury, Cheshire WA4 4AD, United Kingdom \\ ${ }^{7}$ Institute of Physics, Chinese Academy of Sciences, Beijing 100190, China
}

(Received 19 January 2021; revised 1 April 2021; accepted 2 April 2021; published 23 April 2021)

\begin{abstract}
Laser-wakefield acceleration has been demonstrated to be a promising technique for compact electron accelerators. However, it is still challenging to achieve high-quality $100-\mathrm{MeV}$ electron bunches of subfemtosecond duration with current techniques. Here, we present and numerically demonstrate an efficient scheme to produce such high-energy tunable ultrashort electron bunches, which is achieved by the use of a nonlinear wakefield driven by a terawatt few-cycle laser pulse in a tailored plasma channel. With the driving laser pulse energy of $25 \mathrm{~mJ}$ only, the resulting electron bunch can reach $101 \mathrm{MeV}$ with 7.8-pC charge, approximately 4 mrad divergence, approximately 3\% energy spread, energy efficiency above 3\%, and a duration of hundreds of attoseconds. As such laser pulses may be obtained with a kilohertz repetition rate and high stability, our scheme could be interesting for ultrafast science and accelerator community.
\end{abstract}

DOI: 10.1103/PhysRevApplied.15.044039

Electron accelerators are crucial tools to promote scientific and technological advances [1-3], which can benefit greatly from the availability of compact accelerator technologies. Laser-wakefield acceleration (LWFA) [4-7] is an emerging acceleration technique that can produce $100-\mathrm{MeV}[8-10]$ to few-GeV $[11,12]$ electrons in a tabletop scale due to its ultrahigh accelerating gradient on the range of $\mathrm{GV} / \mathrm{cm}$, which is more than 3 orders of magnitude higher than those achievable in conventional radio-frequency accelerators. However, current LWFAs normally rely on Joule-class and multicycle pulsed-laser systems operating at a few- $\mathrm{Hz}$ repetition rate, thereby restraining their dissemination and applications. Terawatt (TW) few-cycle laser pulses typically have multimillijoule (multi-mJ) energies that can operate at a $\mathrm{kHz}$ repetition rate [13]. There is an increasing interest in developing LWFAs with such TW lasers, which may exploit unique abilities for applications, such as acceleration of ultrashort electron bunches and coherent synchrotron radiation by subcycle

\footnotetext{
*xinglong.zhu@sjtu.edu.cn

${ }^{\dagger}$ zmsheng@sjtu.edu.cn

¥These authors contributed equally to this work.
}

laser pulses [14,15], stable $\mathrm{kHz} \mathrm{MeV}$ electron sources by near-single-cycle laser pulses [16], etc.

Over the past decade, there have been considerable efforts to address this quest for electron acceleration using high-repetition-rate multi-mJ lasers interacting with solid targets [17], gas targets [18-20], or liquid targets [21]. However, the energies of the accelerated electrons are limited to the $100 \mathrm{keV}$ to $3 \mathrm{MeV}$ range. Moreover, the beam divergence (hundreds of mrad) and energy spread (on the order of $100 \%$ ) are usually relatively large. There is no practicable way to focus and transport the electron beam with such a large divergence and a broad energy spread, making it hard for practical applications. Recently progresses have been made in $\mathrm{kHz}$ LWFAs using nearsingle-cycle laser pulses in relatively high-density $\left(n_{e} \gtrsim\right.$ $10^{20} \mathrm{~cm}^{-3}$ ) plasmas [16], where electron beams have been produced with approximately 1-pC charge, divergences of approximately 45-mrad FWHM, and energy peaks at approximately $5 \mathrm{MeV}$ but still with large energy spreads up to $60 \%$. There is also unavoidable physical limitation on the energy gain of electrons accelerated in the blowout or bubble regime [22,23] $\Delta E \propto a_{0} n_{c} m_{e} c^{2} / n_{e}<10 \mathrm{MeV}$ due to the dephasing length limited in the $100-\mu \mathrm{m}$ range, where $n_{c}=m_{e} \omega_{0}^{2} / 4 \pi e^{2}$ is the critical plasma density, $e$ and $m_{e}$ 
are, respectively, the electron unit charge and mass, and $a_{0}$ and $\omega_{0}$ are, respectively, the laser amplitude and frequency. On the other side, these methods rely on either the ionization injection or the self-injection, but both injection mechanisms are highly sensitive to laser parameters such as the carrier-envelope phase (CEP) and laser intensity, making electron injection and acceleration difficult to control. More recent studies have shown that the stability of electron injection can be greatly enhanced by using a density gradient [24,25], but it is still difficult to achieve stable electron acceleration with high-charge and low-energy spreads at a few percent level and to increase electron energy to reach the $100-\mathrm{MeV}$ level while maintaining a high stability. In addition, it remains challenging in the generation of attosecond electron bunches from LWFAs, which should be interesting for attosecond physics and ultrafast applications [26,27]. In the previous studies, significant efforts have been made to produce attosecond electron sources, but they mostly either have a very large divergence of hundreds of mrad $[28,29]$ or have a low energy of multi-MeV [30,31]. There is no practicable method proposed to produce high-quality $100-\mathrm{MeV}$ attosecond electron sources.

In this work we propose a scheme to solve these problems, which can produce high-quality subfemtosecond (sub-fs) electron bunches at the $100-\mathrm{MeV}$ level driven by a TW few-cycle laser pulse in a density-tailored plasma channel. The produced bunches show desirable properties and their durations can be as short as a sub-fs (or attosecond) scale. This potentially makes it possible to generate attosecond light pulses in the hard x-ray or even $\gamma$-ray range. The scheme may be realized with existing compact $\mathrm{kHz}$ laser systems, enabling it to achieve stable electron acceleration at a high repetition rate for various applications.

Figure 1 illustrates the schematic diagram of our scheme. A nonlinear plasma wake is excited by a relativistic laser pulse propagating in an underdense plasma. Here a plasma channel is used to efficiently guide the laser pulses and hence the electrons can be accelerated over many diffraction lengths [32-36]. We demonstrate this scheme using three-dimensional (3D) relativistic particlein-cell (PIC) simulations with the EPOCH code [37] that has been equipped with the NDFX scheme [38] for solving Maxwell's equations. The simulation box has a size of $60 \lambda_{0}(x) \times 26 \lambda_{0}(y) \times 26 \lambda_{0}(z)$ with $2400 \times 156 \times 156$ grid cells, which moves at the speed of light $c$ along the $x$ direction, where two macroparticles per cell are used for both electrons and ion species (or protons) and absorbing boundary conditions are employed for both particles and electromagnetic fields. The linearly polarized laser pulse has a spatial-temporal profile of

$$
\mathbf{a}=a_{0} \exp \left(-\frac{r^{2}}{w_{0}^{2}}\right) \sin ^{2}\left(\pi t / 2 \tau_{0}\right) \mathbf{e}_{y},
$$

with the normalized amplitude $a_{0}=1.8$, spot size $w_{0}=$ $6 \lambda_{0}$, wavelength $\lambda_{0}=1 \mu \mathrm{m}$, oscillation period $T_{0}=$ $2 \pi / \omega_{0}$, and pulse duration $\tau_{0}=10 \mathrm{fs}$ (FWHM), which corresponds to the peak intensity of $4.4 \times 10^{18} \mathrm{~W} / \mathrm{cm}^{2}$, peak
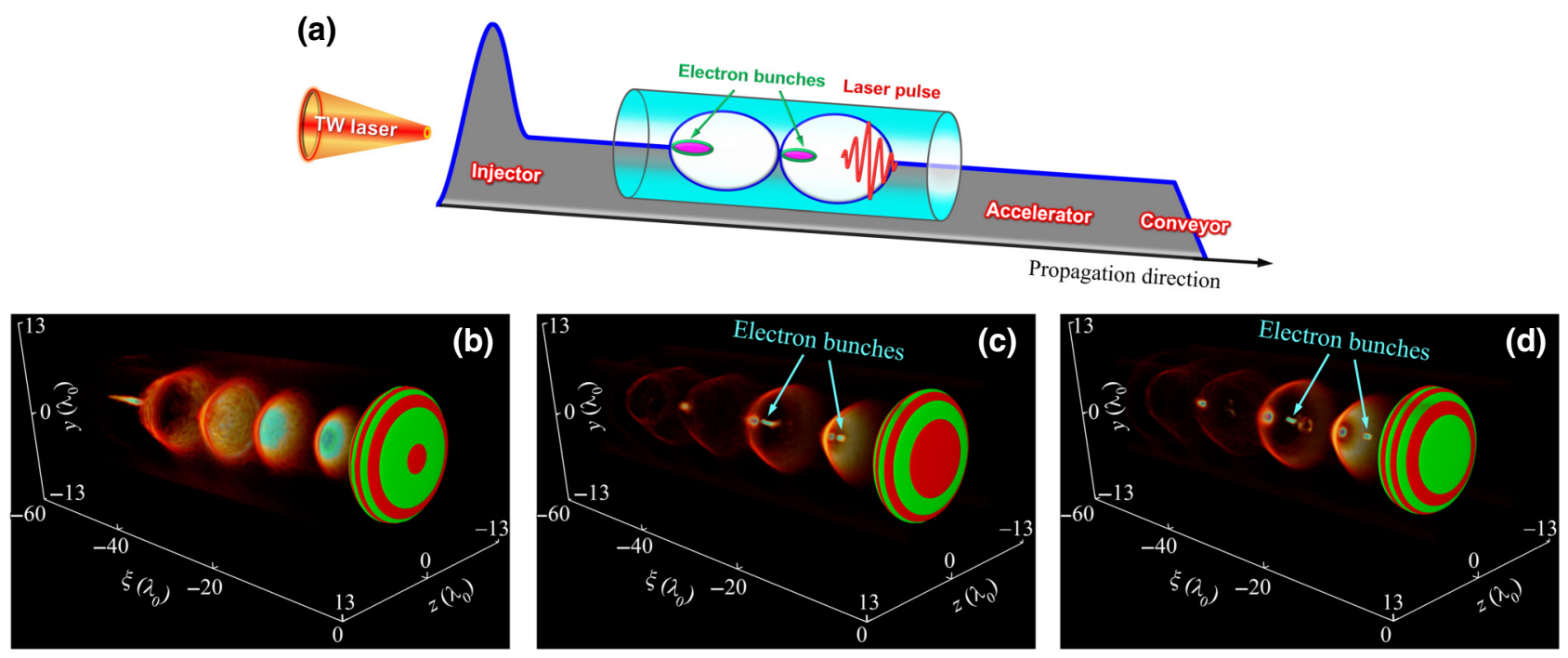

FIG. 1. Schematic illustration. (a) A relativistic TW-class laser pulse propagates in a density-tailored plasma channel with three segments for injection, acceleration, and transportation, respectively. In the first segment, some plasma electrons at the rear of the first few buckets are trapped via the density gradient injection, and are then accelerated by the strong wakefield to high energies in the following accelerator segment. In the final conveyor segment, the accelerated electrons are transported from the plasma to the vacuum. (b)-(d) Illustration of 3D views of the TW laser-driven wakefield accelerator using 3D PIC simulations, which correspond to snapshots shown in Figs. 2(b)-2(d) and more details are given correspondingly. 
power of $2.5 \mathrm{TW}$, and pulse energy of $25 \mathrm{~mJ}$. Such laser pulses are readily available at existing $\mathrm{kHz}$ laser systems. The plasma channel has a transverse parabolic density profile of $n_{e}=n_{0}+\Delta n_{0}$ with a 1-mm longitudinal length, where $n_{0}$ is the on-axis density, $\Delta n_{0}=\left(\lambda_{0}^{2} / \pi^{2} w_{0}^{4}\right) r_{\mathrm{ch}}^{2} n_{c}$ is the channel depth, and $r_{\mathrm{ch}}$ is the channel radius. The longitudinal density structure of the plasma includes three different segments: in the first segment the density bump at the entrance of the plasma acts as an injector via the density-transition (or shock-front) mechanism, the following plasma segment with a lower density works as an accelerator, and the falling slope at the end of the plasma serves as a conveyor for extracting the electrons from the plasma. Here the density bump can be produced by inserting a blade placed at the entrance of a supersonic gas nozzle [39]. After that the density channel can be formed by using a picosecond laser prepulse technique [40]. According to the density-transition injection method $[39,41]$, the on-axis density profile of the injector is set to

$$
\begin{aligned}
n_{e}(x)= & \Theta\left(\frac{L_{1}}{2}-\kappa_{1}\right) \frac{n_{e 1}}{2}\left[\sin \left(\frac{\pi \kappa_{1}}{L_{1}}\right)+1\right] \\
& +\Theta\left(\kappa_{2}-\frac{L_{2}}{2}\right) \Theta\left(\frac{L_{2}}{2}-\kappa_{3}\right) \frac{n_{e 1}}{2} \\
& \times\left[\sin \left(\frac{\pi \kappa_{2}}{L_{2}}\right)+1\right]+\Theta\left(\kappa_{2}-\frac{L_{2}}{2}\right) \\
& \times \Theta\left(\frac{L_{2}}{2}-\kappa_{3}\right) \frac{n_{e 2}}{2}\left[\sin \left(\frac{\pi \kappa_{3}}{L_{2}}\right)+1\right],
\end{aligned}
$$

as shown in Fig. 2(a), where $\Theta(x)$ is the step function, $\kappa_{1}=x-L_{1} / 2, \kappa_{2}=x-L_{1}+L_{2} / 2, \kappa_{3}=x-L_{1}-L_{2} / 2$, $L_{1}=60 \lambda_{0}, L_{2}=35 \lambda_{0}, n_{e 1}=0.009 n_{c}$, and $n_{e 2}=n_{e 1} / 2$, respectively. The scale length of the falling slope is $50 \lambda_{0}$.

Figures 2(b)-2(d) illustrates the evolution of the laser field, plasma density, and wakefield at different positions. At the beginning of the interaction, the electrons cannot catch up with the plasma wake and thus cannot be injected and accelerated. This is mainly attributed to the high phase velocity of the wake, which can be estimated by assuming the local phase velocity of the plasma wave during a density transition, where the laser group velocity is considered as $v_{\mathrm{g}} \simeq c$ since its change induced by a slow variation in the density is small in underdense plasmas [1]. Thus the local phase velocity of the plasma wake can be given by

$$
\beta_{\mathrm{ph}}=\left[1+\left(\xi / k_{p}\right) d k_{p} / d x\right]^{-1},
$$

where $d k_{p} / d x=\left(k_{p} / 2 n_{e}\right) d n_{e} / d x$. As the light-frame coordinate is $\xi=x-c t<0$ behind the laser driver, one has $\beta_{\mathrm{ph}} \approx 1$ for the laser propagation in underdense plasmas with a uniform density profile $d n_{e} / d x \approx 0 ; \beta_{\mathrm{ph}}>1$ at a density up ramp $d n_{e} / d x>0$; and $\beta_{\mathrm{ph}}<1$ at a density down ramp $d n_{e} / d x<0$. In consequence, when the laser drives the plasma wake through the density down ramp, the electron injection will occur due to a rapid decrease of the plasma-wave phase velocity $[42,43]$. The corresponding plasma wavelength $\lambda_{p} \approx 2 \pi c / \omega_{p}$ increases substantially, where $\omega_{p}=\sqrt{4 \pi e^{2} n_{e} / m_{e}}$ is the plasma frequency. As shown in Figs. 2(b) and 2(c), the size of plasma buckets enlarges remarkably after the down-ramp density transition, i.e., $\lambda_{p, 2}>\lambda_{p, 1}$ for the first bucket in the wake. Consequently, considerable electrons move faster than the local-wave phase velocity, so that they are suddenly injected into the accelerating phase of the wakefield and then be trapped and accelerated. Figure 2(e) exhibits the trajectories of some typical electrons accelerated in the first bucket, where the injection occurs rapidly during this density transition, which is quite different from that in the self-injection mechanism.

We now discuss the physics of this acceleration scheme. The phase velocity can be further expressed as $\beta_{\mathrm{ph}, m}=$ $\left[1+m d \lambda_{p} / d x\right]^{-1}$ for these plasma wave buckets with $\xi=$ $-m \lambda_{p}$ behind the laser driver, where $m$ is the serial number of the plasma buckets behind the laser pulse. In order to trap the electrons into plasma buckets, the minimum energy necessary is $\gamma_{\min } \approx \beta_{\mathrm{ph}} / 2 \Delta \phi+\Delta \phi /\left(1+\beta_{\mathrm{ph}}\right)$ in the limit $\gamma_{\mathrm{p}} \Delta \phi \gg 1$ [44], where $\gamma_{\mathrm{p}}=\left(1-\beta_{\mathrm{ph}}^{2}\right)^{-1 / 2}$, $\Delta \phi=\phi_{\max }-\phi_{\min }$, and the normalized wakefield potential $\phi=e \Phi / m_{e} c^{2}$ is assumed to oscillate in the range $\phi_{\min } \leq \phi \leq \phi_{\max }$ periodically with a nonlinear plasma wavelength. A low $\beta_{\mathrm{ph}}$ requires a low injection threshold for trapping the electrons, which results in more electrons trapped in the second bucket than the first bucket since $\beta_{\mathrm{ph}, 2}<\beta_{\mathrm{ph}, 1}$. The third bucket has an even lower $\beta_{\text {ph. }}$. However, its wakefield strength is so much lower (i.e., $\phi_{3}<\phi_{2} \approx \phi_{1}$ ) that only a small number of electrons can be trapped. These injected electrons are then accelerated by the wakefield to high energies until they enter the decelerating (or dephasing) region. Here, we consider only the electrons accelerated from the first bucket unless otherwise stated, since the electrons trapped in the following second and third buckets dephase very quickly and also defocus quickly [see Fig. 2(d)]. On the other hand, to reduce the broad energy component of electron beams in the following buckets, a promising way is to utilize a density-tailored plasma with a shock front at the end of the plasma structure [45]. This will be studied in the near future, where we can place another obstacle at the exit of the gas jet to form such a shock front.

Compared to the previous $\mathrm{kHz}$ LWFA methods with typical $100-\mu$ m-level acceleration lengths, the length $(L \propto$ $\left.c \tau n_{c} / n_{0}\right)$ is dramatically increased to reach nearly $1 \mathrm{~mm}$ in our scheme. This leads to electron acceleration by the intense wakefield $\left(E_{x}>1 \mathrm{GV} / \mathrm{cm}\right)$ to high energies reaching up to the $100-\mathrm{MeV}$ level, as shown in Fig. 3. The energy of trapped electrons increases with the acceleration length $\left(L_{a}=c t\right)$ until they enter the deceleration area as the energy gain relation of $\Delta E=e E_{x} L_{a}$, where the 

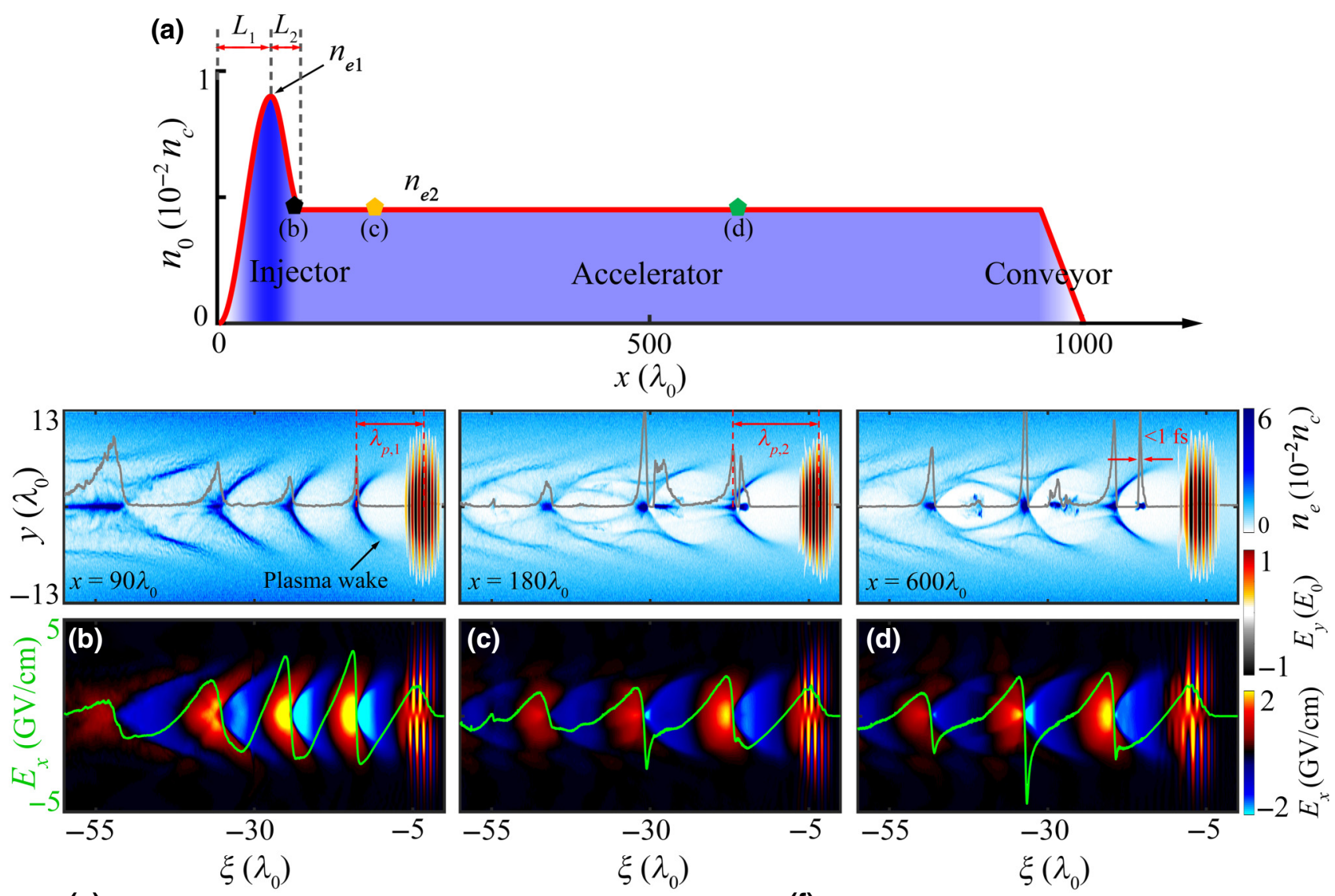

$$
\xi\left(\lambda_{0}\right)
$$
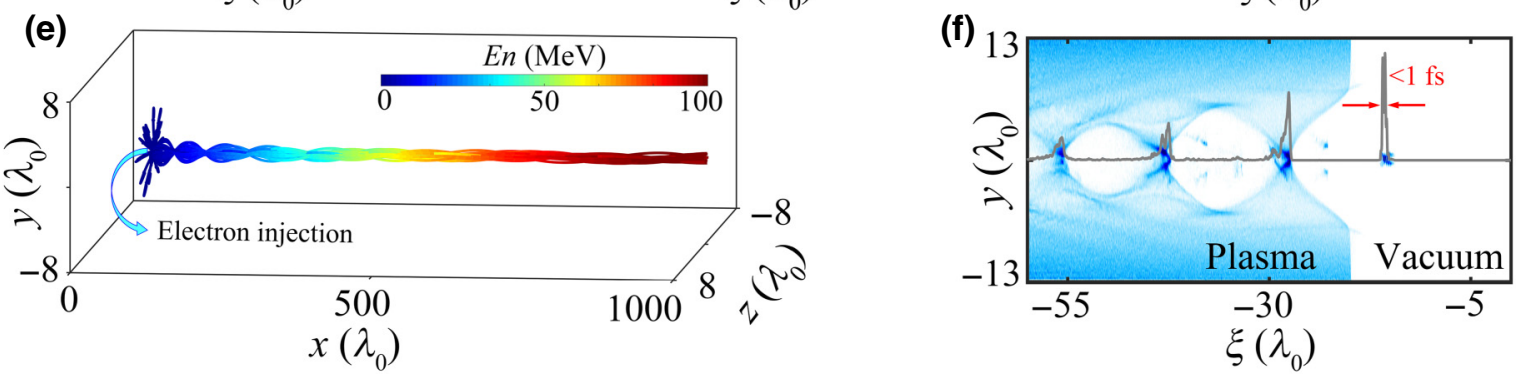

FIG. 2. (a) On-axis density distribution of the plasma channel. The color dots in (a) represent different positions shown in [(b), black dot], [(c), yellow dot], and [(d), green dot]. The plasma density $\left(n_{e}\right)$ and the transverse laser field $\left(E_{y}\right)$ in the upper graphs, the longitudinal wakefield $\left(E_{x}\right)$ and its corresponding on-axis distribution in the lower graphs are shown at different positions $x=90 \lambda_{0}$ (b), $180 \lambda_{0}(\mathrm{c})$, and $600 \lambda_{0}$ (d). The density and laser field are normalized to $n_{c}$ and $E_{0}=m_{e} c \omega_{0} / e$, respectively. (e) The trajectories of some typical electrons accelerated in the first bucket. (f) Snapshot of the electron bunches transported from the plasma to the vacuum.

accelerating field of the wake can be estimated by $E_{x} \simeq$ $E_{x}^{m}\left\{1-\left[\left(c-v_{\phi}\right) / R_{b}\right] t\right\}, E_{x}^{m}=\sqrt{a_{0}} m_{e} c \omega_{p} / e$ is the peak amplitude, $v_{\phi} \simeq c\left[1-3 \omega_{p}^{2} / 2 \omega_{0}^{2}\right]$ is the phase velocity in the underdense plasma limit $\omega_{p}^{2} \ll \omega_{0}^{2}$, the radius of the wake cavity is approximately $R_{b} \simeq 2 \sqrt{a_{0}} / k_{p}$. Thus one can obtain

$$
\Delta E \simeq e E_{x}^{m} c t\left(1-\frac{c-v_{\phi}}{R_{b}} t\right),
$$

which predicts the scaling of the electron energy with interaction time $t$, as seen in Fig. 3(b). At the time $t=1000 T_{0}$, the increase of the energy gradually slows down, because the injected electrons nearly arrive at the dephasing region in the first bucket. Besides, when the drive laser propagates in a longer plasma, the effects of laser redshift, depletion, and diffraction become considerable and thus obviously reduce the pulse energy, which impede electron acceleration. Therefore, taking an appropriate plasma length of about $1 \mathrm{~mm}$ is beneficial for high-quality electron acceleration. The resulting electron beam shows the peak energy of $101 \mathrm{MeV}$ and the conversion efficiency of $3.1 \%$ from the laser pulse to the quasimonoenergetic electron bunch accelerated in the first bucket. Finally, the produced bunch 

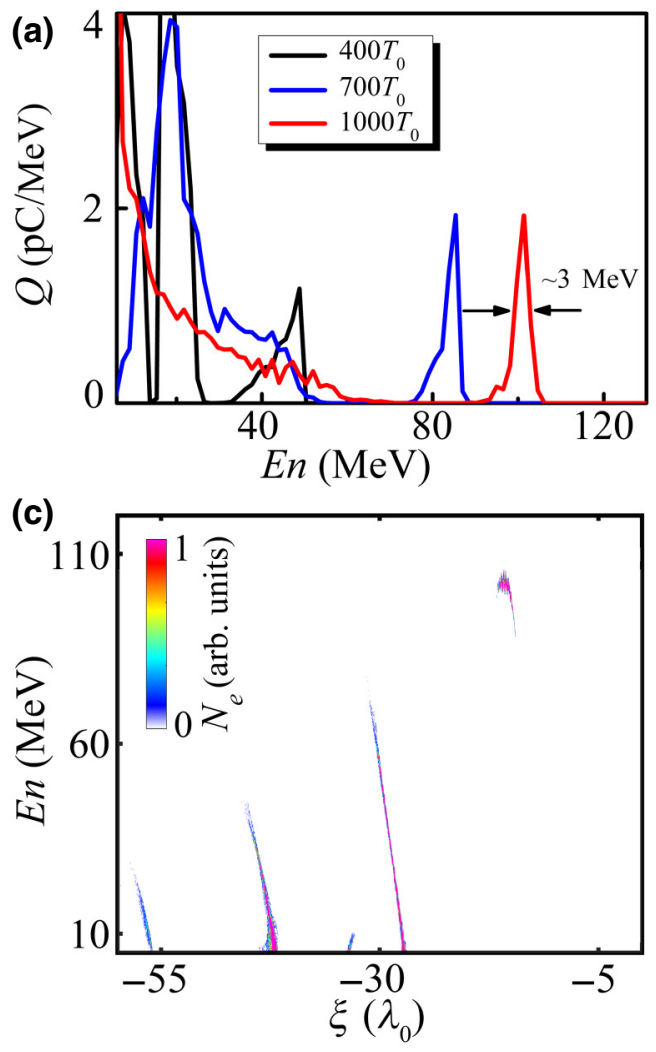

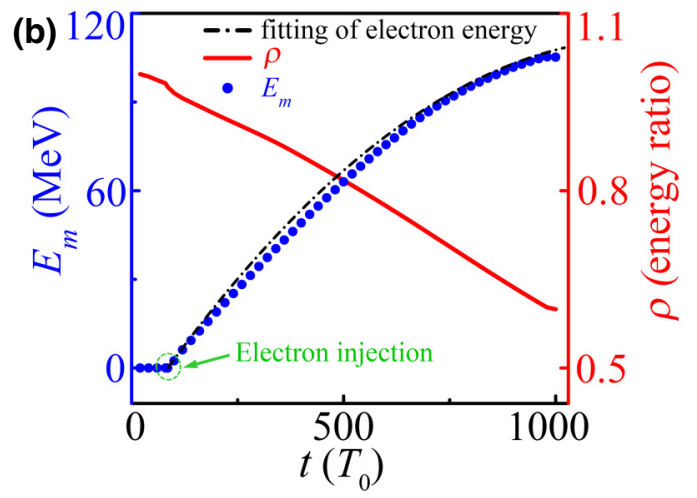

(d)

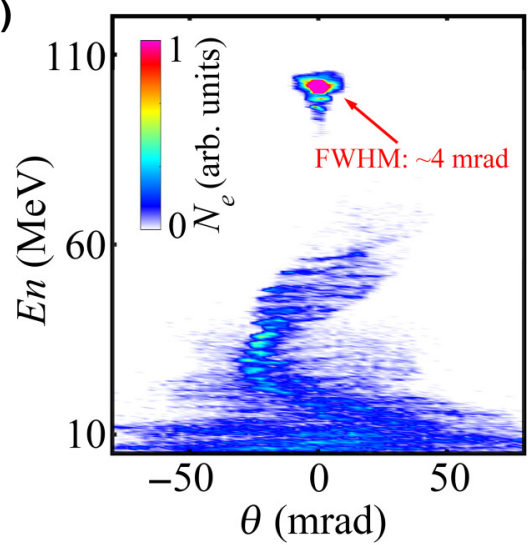

FIG. 3. (a) Energy spectra of the accelerated electrons at different times. (b) The maximum energy $\left(E_{m}\right)$ of the accelerated electrons and the ratio (defined as $\left.\rho=E_{l} / E_{l 0}\right)$ of the remaining laser pulse energy $\left(E_{l}\right)$ to the initial pulse energy $\left(E_{l 0}\right)$, as a function of the interaction time $t$. The dashed curve in (b) indicates the fitting of electron energy as suggested by Eq. (2). The distributions of accelerated electrons in energy-longitudinal space (c) and energy angle (d). has a 7.8-pC charge with a low divergence of approximately $4 \mathrm{mrad}$, a narrow energy spread of approximately $3 \%$, and a short-bunch duration of $<1 \mathrm{fs}$.

The charge, energy efficiency, and duration of the generated electron beams can be tuned by changing the plasma parameters. Here we mainly investigate the effect of the injector segment on the electron beams, because it plays a more useful role on injection, trapping and beam properties of the electrons in the wakefield as compared with the accelerator segment that is primarily responsible for accelerating the trapped electrons to high energies. Additional simulations show that, when the scale length of the falling slope at the end of the plasma is within the range of $100 \mu \mathrm{m}$, there is no significant effect on the properties of electron beams transported out of the plasma. Otherwise, the trapped electrons will enter the dephasing region and consequently decrease their energy during propagation in the falling slope of the plasma. Figure 4(a) shows the effect of the density down-ramp length $L_{2}$ on the accelerated electrons. It is shown that a moderately short length is beneficial for electron acceleration with higher charges and energy efficiencies. This is attributed to a decrease in the wave-breaking time [46] $t_{\mathrm{br}}=2\left(L_{i}+x_{0}\right) / c$ in the relativistic limit, since the scale length of the density inhomogeneity $L_{i}=L_{2}\left(n_{e 1}+n_{e 2}\right) / 2\left(n_{e 1}-n_{e 2}\right)$ decreases when using a shorter $L_{2}$. This leads to an increase in the probability of the injection and thus more electrons can be trapped into the plasma wake and be accelerated, where the electron charge can be approximately scaled as $Q \propto 1 / L_{2}$, as seen by the dashed line in Fig. 4(a). It is noted that the electron bunches have a low charge when taking a long down ramp (e.g., $L_{2}=45 \lambda_{0}$ ), however, the bunch generated in the first bucket has a short duration of about 800 as. Moreover, the increase of the inhomogeneity length may cause the injected electrons to be closer to the tail of the wakefield and thus increase their peak energy. Such ultrashort relativistic electron bunches are useful for attosecond $\mathrm{x}-\mathrm{\gamma}$-ray pulse generation and many ultrafast studies [26-31].

Figure 4(b) presents the dependence of electron acceleration on the plasma density $n_{e 1}$ in the injection segment. One can see that a moderately high-density plasma is beneficial to inject more electrons into the wake, where both the charge and energy efficiency of the electrons increase with $n_{e 1}$. This is because the density gradient becomes sharp with a small $\beta_{\text {ph }}$, which gives rise to a decrease in the inhomogeneity length $L_{i}$ and so a decrease in the breaking time $t_{\mathrm{br}}$, thereby causing a low threshold for electron trapping in the wake wave. One may find that the charge of the trapped electrons scales as $Q \propto n_{e 1}$ from numerical simulations. For example, when using $n_{e 1}=$ $0.012 n_{c}$, the electrons produced in the first bucket have a surprisingly high charge of as high as $34 \mathrm{pC}$ and ultrahigh energy conversion efficiency reaching $8.5 \%$, both of them are more than tens of times higher than those generated in the kHz multi-mJ LWFAs [16-21]. Furthermore, 
(a)
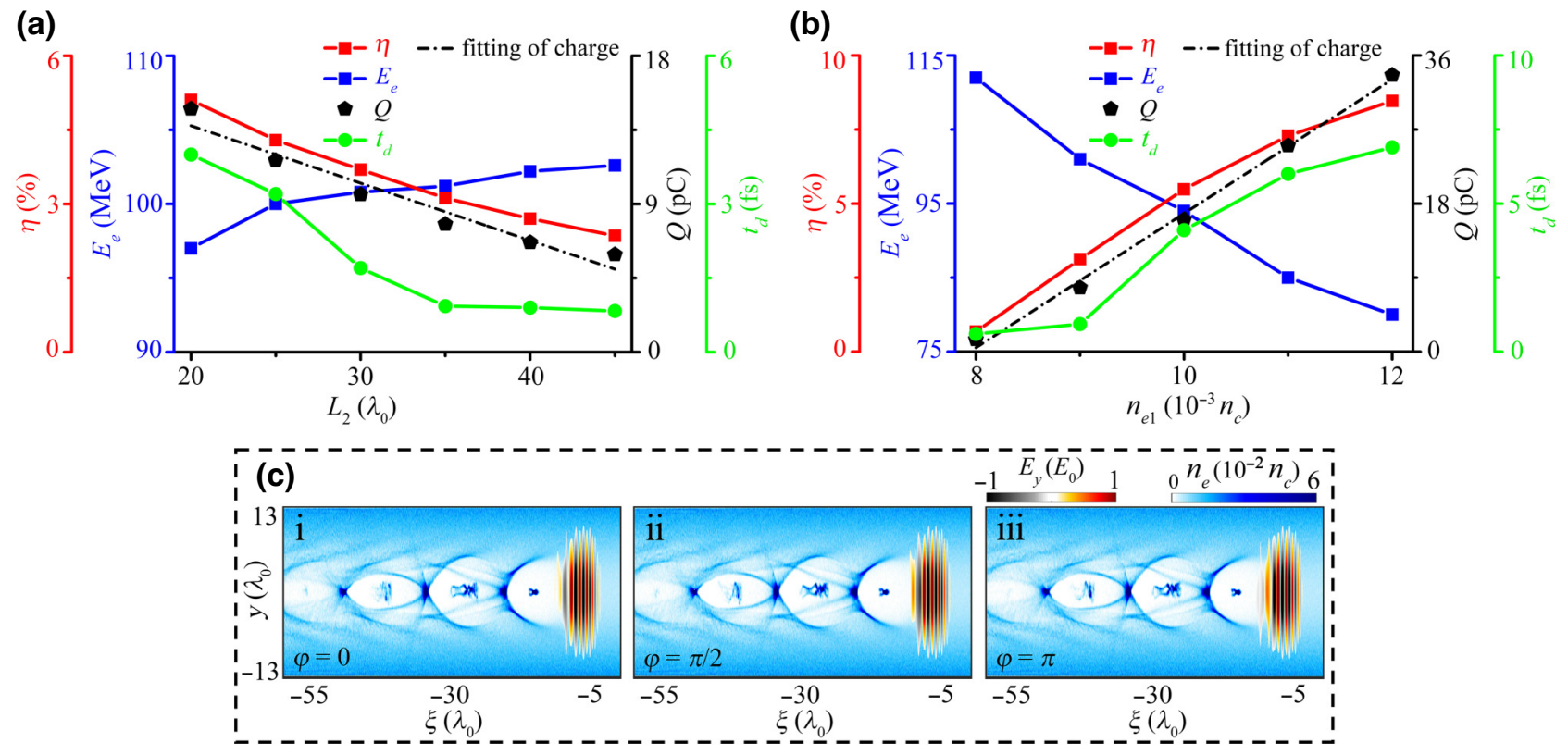

FIG. 4. Energy efficiency $(\eta)$, peak energy $\left(E_{e}\right)$, charge $(Q)$, and beam duration $\left(t_{d}\right)$ of the electrons accelerated in the first bucket as functions of (a) the length $L_{2}$ of the plasma density down ramp between the injector and accelerator, and (b) the density $n_{e 1}$ in the injection segment. The dashed lines in (a) and (b), respectively, indicate the $Q \propto 1 / L_{2}$ fitting and the $Q \propto n_{e 1}$ fitting for the total charge of the electrons injected in the first bucket. (c) Distributions of the plasma density and the transverse electric field for three cases with different initial laser phases $\varphi=0$ (i), $\pi / 2$ (ii), and $\pi$ (iii) at the same position $x=750 \lambda_{0}$, where all other parameters are the same as those shown in Fig. 2.

it is interesting to note that the durations of the electron bunches decrease significantly when using a low density (e.g., $n_{e 1}=0.008 n_{c}$ ), which can be as short as about 600 as in the first bucket, making them suitable for applications requiring attosecond-scale electron bunches. The peak energy of the accelerated electrons increases with reducing $n_{e 1}$ since they can be injected closer to the tail of the wake.
To examine the effects of laser parameters on our scheme, we investigate the drive pulse with three different initial phases (or CEPs), while keeping all other parameters unchanged. Simulation results indicate that the initial laser phase has no obvious effects on this acceleration scheme, where the produced electron bunches have nearly the same beam quality, energy, and charge even for pulse propagation over a 750- $\mu \mathrm{m}$ distance, as shown in Fig. 4(c). (a)

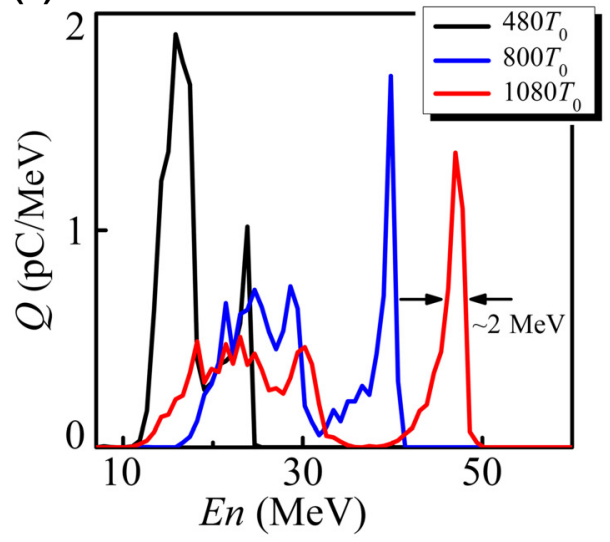

(b)

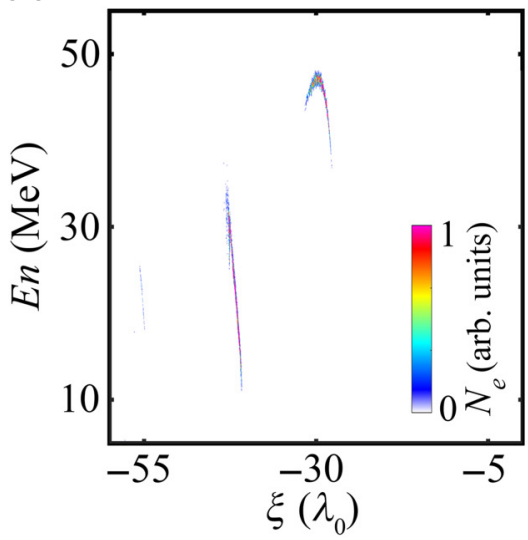

(c)

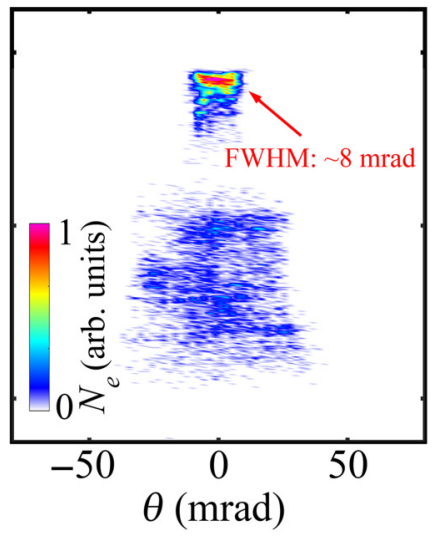

FIG. 5. (a) Electron energy spectra at different times. The energy-space (b) and energy-angular (c) distributions of the electrons generated from a $10-\mathrm{mJ}$ LWFA. 
Furthermore, the electron injection can be controlled by using a density transition and the laser pulse can be steadily propagated through a $1-\mathrm{mm}$-scale plasma channel without significant diffraction, strong redshifting, or nonlinear effects. These make it feasible to achieve high-quality electron acceleration with high charge and high energy at a high stability.

To further demonstrate the robustness of this scheme, we consider a case with only 10 -mJ laser pulse to drive the electron acceleration. This pulse driver has a same spatial-temporal profile as that shown in Fig. 2, and with $a_{0}=1.5, w_{0}=5 \lambda_{0}$, and $\tau_{0}=8.3 \mathrm{fs}$ (FWHM), which has a peak intensity of $3 \times 10^{18} \mathrm{~W} / \mathrm{cm}^{2}$, peak power of $1.2 \mathrm{TW}$, and pulse energy of $10 \mathrm{~mJ}$. The plasma has a similar profile as that shown in Fig. 2, while other parameters are adjusted accordingly with $L_{2}=20 \lambda_{0}, n_{e 1}=0.007 n_{c}$, and $n_{e 2}=n_{e 1} / 2$. Figure 5 shows the resulting electron bunch has an approximately 3.8-pC charge and a high energy peak at $47 \mathrm{MeV}$ with a small energy spread of approximately $4 \%$ and approximately $8 \mathrm{mrad}$ low divergence (FWHM). This reveals that our scheme is robust to drive high-quality high-repetition-rate electron acceleration, which not only can operate at existing box-sized $\mathrm{kHz}$ laser systems, but also has high charge and high energy with high beam quality.

In conclusion, we propose and demonstrate, by 3D PIC simulations, an efficient way to produce high-quality electron bunches using a multi-mJ TW-class laser pulse in a tailored plasma channel. The charge, energy, and duration of the electron bunches can be flexibly controlled by choosing the laser plasma parameters. In a typical case with the driving laser energy of $25 \mathrm{~mJ}$, the electron bunches are accelerated to beyond $100 \mathrm{MeV}$ with a duration as short as a few hundred attoseconds, low divergences of approximately $4 \mathrm{mrad}$, narrow energy spreads of approximately $3 \%$, large charge of tens of $\mathrm{pC}$ and high energy efficiency above $3 \%$. Our work suggests a promising way for compact relativistic $\mathrm{kHz}$ electron sources for a variety of applications, such as ultrashort x-ray generation $[2,47,48]$, compact free-electron lasers $[49,50]$, and electron injectors for $\mathrm{GeV}$ particle accelerators [51].

\section{ACKNOWLEDGMENTS}

This work is supported by the National Key Research and Development Program of China (Grant No. 2018YFA 0404802), the Strategic Priority Research Program of Chinese Academy of Sciences (Grant No. XDA25050100), the National Natural Science Foundation of China (Grants No. 11991074, No. 11775144, and No. 11975154), the Grant from the Office of Science and Technology, Shanghai Municipal Government (No. 18JC1410700), EC H2020 EuPRAXIA (No. 653782), UK STFC Cockcroft Institute Core Grant (No. ST/P002056/1), and Science Challenge Project (No. TZ2018005). The development of
EPOCH code is supported in part by the UK EPSRC (Grant No. EP/G056803/1). Simulations are carried out on the PI supercomputer at Shanghai Jiao Tong University and the ARCHER supercomputer in Edinburgh. Access to ARCHER is via the Plasma HEC Consortium supported by EPSRC (Grants No. EP/L000237/1 and No. EP/R029148/1).

[1] E. Esarey, C. Schroeder, and W. Leemans, Physics of laserdriven plasma-based electron accelerators, Rev. Mod. Phys. 81, 1229 (2009).

[2] S. Corde, K. Ta Phuoc, G. Lambert, R. Fitour, V. Malka, A. Rousse, A. Beck, and E. Lefebvre, Femtosecond $\mathrm{x}$ rays from laser-plasma accelerators, Rev. Mod. Phys. 85, 1 (2013).

[3] C. Bostedt, S. Boutet, D. M. Fritz, Z. Huang, H. J. Lee, H. T. Lemke, A. Robert, W. F. Schlotter, J. J. Turner, and G. J. Williams, Linac coherent light source: The first five years, Rev. Mod. Phys. 88, 015007 (2016).

[4] T. Tajima and J. Dawson, Laser Electron Accelerator, Phys. Rev. Lett. 43, 267 (1979).

[5] K. Nakajima, D. Fisher, T. Kawakubo, H. Nakanishi, A. Ogata, Y. Kato, Y. Kitagawa, R. Kodama, K. Mima, H. Shiraga, et al., Observation of Ultrahigh Gradient Electron Acceleration by a Self-Modulated Intense Short Laser Pulse, Phys. Rev. Lett. 74, 4428 (1995).

[6] V. Malka, J. Faure, Y. A. Gauduel, E. Lefebvre, A. Rousse, and K. T. Phuoc, Principles and applications of compact laser-plasma accelerators, Nat. Phys. 4, 447 (2008).

[7] S. M. Hooker, Developments in laser-driven plasma accelerators, Nat. Photon. 7, 775 (2013).

[8] S. P. D. Mangles, C. D. Murphy, Z. Najmudin, A. G. R. Thomas, J. L. Collier, A. E. Dangor, E. J. Divall, P. S. Foster, J. G. Gallacher, C. J. Hooker, et al., Monoenergetic beams of relativistic electrons from intense laser-plasma interactions, Nature 431, 535 (2004).

[9] C. G. Geddes, C. S. Toth, J. Van Tilborg, E. Esarey, C. B. Schroeder, D. Bruhwiler, C. Nieter, J. Cary, and W. P. Leemans, High-quality electron beams from a laser wakefield accelerator using plasma-channel guiding, Nature 431, 538 (2004).

[10] J. Faure, Y. Glinec, A. Pukhov, S. Kiselev, S. Gordienko, E. Lefebvre, J.-P. Rousseau, F. Burgy, and V. Malka, A laser-plasma accelerator producing monoenergetic electron beams, Nature 431, 541 (2004).

[11] X. Wang, R. Zgadzaj, N. Fazel, Z. Li, S. A. Yi, X. Zhang, W. Henderson, Y. Y. Chang, R. Korzekwa, H. E. Tsai, et al., Quasi-monoenergetic laser-plasma acceleration of electrons to $2 \mathrm{GeV}$, Nat. Commun. 4, 1988 (2013).

[12] A. J. Gonsalves, K. Nakamura, J. Daniels, C. Benedetti, C. Pieronek, T. C. H. de Raadt, S. Steinke, J. H. Bin, S. S. Bulanov, J. van Tilborg, et al., Petawatt Laser Guiding and Electron Beam Acceleration to $8 \mathrm{GeV}$ in a LaserHeated Capillary Discharge Waveguide, Phys. Rev. Lett. 122, 084801 (2019).

[13] R. Budriūnas, T. Stanislauskas, J. Adamonis, A. Aleknavičius, G. Veitas, D. Gadonas, S. Balickas, A. Michailovas, and A. Varanavičius, $53 \mathrm{w}$ average power CEP-stabilized 
OPCPA system delivering 5.5 TW few cycle pulses at $1 \mathrm{kHz}$ repetition rate, Opt. Express 25, 5797 (2017).

[14] B. Rau, T. Tajima, and H. Hojo, Coherent Electron Acceleration by Subcycle Laser Pulses, Phys. Rev. Lett. 78, 3310 (1997).

[15] T. Tajima, X. Q. Yan, and T. Ebisuzaki, Wakefield acceleration, Rev. Mod. Plasma Phys. 4, 7 (2020).

[16] D. Guénot, D. Gustas, A. Vernier, B. Beaurepaire, F. Böhle, M. Bocoum, M. Lozano, A. Jullien, R. Lopez-Martens, A. Lifschitz, et al., Relativistic electron beams driven by $\mathrm{kHz}$ single-cycle light pulses, Nat. Photon. 11, 293 (2017).

[17] A. G. Mordovanakis, J. Easter, N. Naumova, K. Popov, P.-E. Masson-Laborde, B. Hou, I. Sokolov, G. Mourou, I. V. Glazyrin, W. Rozmus, et al., Quasimonoenergetic Electron Beams with Relativistic Energies and Ultrashort Duration From Laser-Solid Interactions at $0.5 \mathrm{kHz}$, Phys. Rev. Lett. 103, 235001 (2009).

[18] Z. H. He, B. Hou, J. A. Nees, J. H. Easter, J. Faure, K. Krushelnick, and A. G. R. Thomas, High repetition-rate wakefield electron source generated by few-millijoule, 30 fs laser pulses on a density downramp, New J. Phys. 15, 053016 (2013).

[19] B. Beaurepaire, A. Vernier, M. Bocoum, F. Böhle, A. Jullien, J. P. Rousseau, T. Lefrou, D. Douillet, G. Iaquaniello, R. Lopez-Martens, et al., Effect of the Laser Wave Front in a Laser-Plasma Accelerator, Phys. Rev. X 5, 031012 (2015).

[20] F. Salehi, A. J. Goers, G. A. Hine, L. Feder, D. Kuk, B. Miao, D. Woodbury, K. Y. Kim, and H. M. Milchberg, Mev electron acceleration at $1 \mathrm{kHz}$ with $<10 \mathrm{~mJ}$ laser pulses, Opt. Lett. 42, 215 (2017).

[21] S. Feister, D. R. Austin, J. T. Morrison, K. D. Frische, C. Orban, G. Ngirmang, A. Handler, J. R. H. Smith, M. Schillaci, J. A. LaVerne, et al., Relativistic electron acceleration by $\mathrm{mJ}$-class $\mathrm{kHz}$ lasers normally incident on liquid targets, Opt. Express 25, 18736 (2017).

[22] A. Pukhov and J. Meyer-ter-Vehn, Laser wake field acceleration: The highly non-linear broken-wave regime, Appl. Phys. B 74, 355 (2002).

[23] W. Lu, C. Huang, M. Zhou, W. B. Mori, and T. Katsouleas, Nonlinear Theory for Relativistic Plasma Wakefields in the Blowout Regime, Phys. Rev. Lett. 96, 165002 (2006).

[24] L. Rovige, J. Huijts, I. Andriyash, A. Vernier, V. Tomkus, V. Girdauskas, G. Raciukaitis, J. Dudutis, V. Stankevic, P. Gecys, et al., Demonstration of stable long-term operation of a kilohertz laser-plasma accelerator, Phys. Rev. Accel. Beams 23, 093401 (2020).

[25] J. Huijts, I. Andriyash, L. Rovige, A. Vernier, and J. Faure, Carrier-envelope phase effects in Laser Wakefield Acceleration with near-single-cycle pulses, e-print arXiv:2006.10566.

[26] F. Krausz and M. Ivanov, Attosecond physics, Rev. Mod. Phys. 81, 163 (2009).

[27] H. C. Wu and J. Meyer-ter-Vehn, Giant half-cycle attosecond pulses, Nat. Photon. 6, 304 (2012).

[28] T. V. Liseykina, S. Pirner, and D. Bauer, Relativistic Attosecond Electron Bunches From Laser-Illuminated Droplets, Phys. Rev. Lett. 104, 095002 (2010).
[29] X.-L. Zhu, M. Chen, T.-P. Yu, S.-M. Weng, L.-X. Hu, P. McKenna, and Z.-M. Sheng, Bright attosecond $\gamma-$ ray pulses from nonlinear compton scattering with laserilluminated compound targets, Appl. Phys. Lett. 112, 174102 (2018).

[30] F. Y. Li, Z. M. Sheng, Y. Liu, J. Meyer-ter-Vehn, W. B. Mori, W. Lu, and J. Zhang, Dense Attosecond Electron Sheets From Laser Wakefields Using an Up-Ramp Density Transition, Phys. Rev. Lett. 110, 135002 (2013).

[31] M. P. Tooley, B. Ersfeld, S. R. Yoffe, A. Noble, E. Brunetti, Z. M. Sheng, M. R. Islam, and D. A. Jaroszynski, Towards Attosecond High-Energy Electron Bunches: Controlling Self-Injection in Laser-Wakefield Accelerators Through Plasma-Density Modulation, Phys. Rev. Lett. 119, 044801 (2017).

[32] C. G. R. Geddes, C. Toth, J. van Tilborg, E. Esarey, C. B. Schroeder, J. Cary, and W. P. Leemans, Guiding of Relativistic Laser Pulses by Preformed Plasma Channels, Phys. Rev. Lett. 95, 145002 (2005).

[33] J. Fuchs, E. d'Humières, Y. Sentoku, P. Antici, S. Atzeni, H. Bandulet, S. Depierreux, C. Labaune, and A. Schiavi, Enhanced Propagation for Relativistic Laser Pulses in Inhomogeneous Plasmas Using Hollow Channels, Phys. Rev. Lett. 105, 225001 (2010).

[34] X. L. Zhu, M. Chen, S. M. Weng, P. McKenna, Z. M. Sheng, and J. Zhang, Single-Cycle Terawatt Twisted-Light Pulses at Midinfrared Wavelengths above $10 \mu \mathrm{m}$, Phys. Rev. Appl. 12, 054024 (2019).

[35] X. L. Zhu, S. M. Weng, M. Chen, Z. M. Sheng, and J. Zhang, Efficient generation of relativistic near-single-cycle mid-infrared pulses in plasmas, Light Sci. Appl. 9, 46 (2020).

[36] X.-L. Zhu, M. Chen, S.-M. Weng, T.-P. Yu, W.-M. Wang, F. He, Z.-M. Sheng, P. McKenna, D. A. Jaroszynski, and J. Zhang, Extremely brilliant $\mathrm{GeV} \gamma$-rays from a two-stage laser-plasma accelerator, Sci. Adv. 6, eaaz 7240 (2020).

[37] T. D. Arber, K. Bennett, C. S. Brady, A. Lawrence-Douglas, M. G. Ramsay, N. J. Sircombe, P. Gillies, R. G. Evans, H. Schmitz, A. R. Bell, et al., Contemporary particlein-cell approach to laser-plasma modelling, Plasma Phys. Controlled Fusion 57, 113001 (2015).

[38] A. Pukhov, Three-dimensional electromagnetic relativistic particle-in-cell code VLPL (Virtual Laser Plasma Lab), J. Plasma Phys. 61, 425 (1999).

[39] A. Buck, J. Wenz, J. Xu, K. Khrennikov, K. Schmid, M. Heigoldt, J. M. Mikhailova, M. Geissler, B. Shen, F. Krausz, et al., Shock-Front Injector for High-Quality Laser-Plasma Acceleration, Phys. Rev. Lett. 110, 185006 (2013).

[40] Y. Mizuta, T. Hosokai, S. Masuda, A. Zhidkov, K. Makito, N. Nakanii, S. Kajino, A. Nishida, M. Kando, M. Mori, et al., Splash plasma channels produced by picosecond laser pulses in argon gas for laser wakefield acceleration, Phys. Rev. ST Accel. Beams 15, 121301 (2012).

[41] A. J. Gonsalves, K. Nakamura, C. Lin, D. Panasenko, S. Shiraishi, T. Sokollik, C. Benedetti, C. B. Schroeder, C. G. R. Geddes, J. van Tilborg, et al., Tunable laser plasma accelerator based on longitudinal density tailoring, Nat. Phys. 7, 862 (2011). 
[42] H. Suk, N. Barov, J. B. Rosenzweig, and E. Esarey, Plasma Electron Trapping and Acceleration in a Plasma Wake Field Using a Density Transition, Phys. Rev. Lett. 86, 1011 (2001).

[43] A. V. Brantov, T. Z. Esirkepov, M. Kando, H. Kotaki, V. Y. Bychenkov, and S. V. Bulanov, Controlled electron injection into the wake wave using plasma density inhomogeneity, Phys. Plasmas 15, 073111 (2008).

[44] E. Esarey and M. Pilloff, Trapping and acceleration in nonlinear plasma waves, Phys. Plasmas 2, 1432 (1995).

[45] A. Döpp, C. Thaury, E. Guillaume, F. Massimo, A. Lifschitz, I. Andriyash, J. P. Goddet, A. Tazfi, K. Ta Phuoc, and V. Malka, Energy-Chirp Compensation in a Laser Wakefield Accelerator, Phys. Rev. Lett. 121, 074802 (2018).

[46] S. Bulanov, N. Naumova, F. Pegoraro, and J. Sakai, Particle injection into the wave acceleration phase due to nonlinear wake wave breaking, Phys. Rev. E 58, R5257 (1998).
[47] H. P. Schlenvoigt, K. Haupt, A. Debus, F. Budde, O. Jackel, S. Pfotenhauer, H. Schwoerer, E. Rohwer, J. G. Gallacher, E. Brunetti, et al., A compact synchrotron radiation source driven by a laser-plasma wakefield accelerator, Nat. Phys. 4, 130 (2008).

[48] M. Fuchs, R. Weingartner, A. Popp, Z. Major, S. Becker, J. Osterhoff, I. Cortrie, B. Zeitler, R. Hörlein, G. D. Tsakiris, et al., Laser-driven soft-X-ray undulator source, Nat. Phys. 5, 826 (2009).

[49] K. Nakajima, Towards a table-top free-electron laser, Nat. Phys. 4, 92 (2008).

[50] A. R. Maier, A. Meseck, S. Reiche, C. B. Schroeder, T. Seggebrock, and F. Grüner, Demonstration Scheme for a Laser-Plasma-Driven Free-Electron Laser, Phys. Rev. X 2, 031019 (2012).

[51] E. Adli, A. Ahuja, O. Apsimon, R. Apsimon, A. M. Bachmann, D. Barrientos, F. Batsch, J. Bauche, V. K. Berglyd Olsen, M. Bernardini, et al., Acceleration of electrons in the plasma wakefield of a proton bunch, Nature 561, 363 (2018). 